\title{
Applications of Payload Directed Flight
}

\author{
Corey Ippolito \\ NASA Ames Research Center \\ Moffett Field, CA 94035 \\ 650-604-1605 \\ corey.a.ippolito@nasa.gov
}

\author{
Matt Fladeland \\ NASA Ames Research Center \\ Moffett Field, CA 94035 \\ 650-604-3325 \\ matthew.m.fladeland@nasa.gov
}

\author{
Yoo Hsiu Yeh \\ Carnegie Mellon University \\ Moffett Field, CA 94035 \\ 650-604-0863 \\ yoo.h.yeh@nasa.gov
}

\begin{abstract}
Next generation aviation flight control concepts require autonomous and intelligent control system architectures that close control loops directly around payload sensors in manner more integrated and cohesive that in traditional autopilot designs. Research into payload directed flight control at NASA Ames Research Center is investigating new and novel architectures that can satisfy the requirements for next generation control and automation concepts for aviation. Tighter integration between sensor and machine requires definition of specific sensor-directed control modes to tie the sensor data directly into a vehicle control structures throughout the entire control architecture, from low-level stability and control loops, to higher level mission planning and scheduling reasoning systems. Payload directed flight systems can thus provide guidance, navigation, and control for vehicle platforms hosting a suite of onboard payload sensors. This paper outlines related research into the field of payload directed flight, and outlines requirements and operating concepts for payload directed flight systems based on identified needs from the scientific literature. ${ }^{12}$
\end{abstract}

\section{TABLE OF CONTENTS}

1. INTRODUCTION . .1

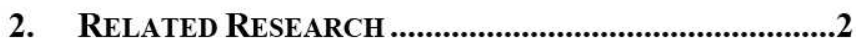

3. GENERAL CONCEPTS OF OPERATIONS .........................4

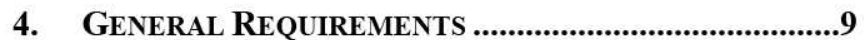

5. CONCLUSION AND FUTURE RESEARCH ........................12

6. REFERENCES .................................................................13

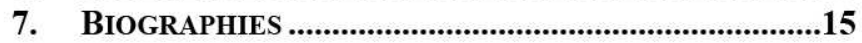

\section{INTRODUCTION}

The enormous benefits of fielding sensor payloads on suborbital flight platforms is being firmly reinforced through the growing number of successful high profile missions involving sensors with ever increasing sophistication and accuracy. In the current practice of fielding advanced sensor payload systems on manned and large unmanned aircraft platforms, automation architectures and mission concepts do not involve explicit autonomous loop closure from the payload sensor data to the autopilot system. However, in the realm of small-scale autonomous aerial vehicle systems, significant technological advances have been made by tying autonomic systems with payload components, especially in

\footnotetext{
${ }^{1}$ U.S. Government work not protected by U.S. copyright

${ }^{2}$ IEEEAC paper \#1410, Version 4, Updated 2008:11:03
}

the milieu of academic research; the results of these innovative research projects (as summarized in Section 2) provide strong arguments for the benefit of sensor-directed loop closure and tight coupling between the autopilot and payload sensor subsystems.

Development of a sensor-integrated vehicle automation architecture on a full-scale manned or unmanned flight platform is inherently a large cross-disciplinary endeavor requiring significant resource and schedule allocation. The engineering approach to such undertakings must be firmly established before the proliferation of such concepts can be realized. The purpose of the Payload Directed Flight (PDF) project at NASA Ames Research Center is to research architectures and methodologies through which subsonic fixed-wing aerial vehicles can meet payload-specific objectives through controllers that close the loop around payload sensors. These architectures must be capable of satisfying mission objectives of the sensor payloads in specific regard to providing observations of partially observable phenomena, such as earth science subjects which are typically large in size and external to the controlled vehicle system. These next generation architectures are envisioned to be payload-centric, closing multiple control loops directly around the output of payload sensors, reconfigurable, able to close the loop on a variety of sensors payloads, both existing and yet to be developed, adaptive, able to respond to change data input from sensor payloads, and intelligent, endowed with limited decision making capabilities that help the aircraft and/or pilot maximize data return from the onboard payloads and remote sensors.

Onboard sensors must be able to relay information beyond raw and filtered sensor data to the control system architecture, such as the quality of data being return, desired targeting locations within the external phenomena, desired target models and tracking filters, constraints that the payload must impart on the vehicle to perform data collection, and higher level mission objectives with regards to the payload. At various layers of control - where each layer is characterized by its operating frequency - the various autonomous and intelligent components must utilize the sensor information and process the data to affect control over the vehicle to meet the requirements of the mission.

This paper outlines the operational concepts and general requirements for payload directed flight system architectures, details a prototypical architecture, and describes applications to an autonomous vehicle system. 
The architecture described allows for sensor data to support operational control modes and functionality throughout the control architecture. A prototypical middle-layer controller system is introduced to satisfy the requirements of payload directed flight. This system produces flight guidance through trajectory generation and path planning, given the requirements, constraints, and objectives of missions directed by sensor payloads.

\section{RELATED RESEARCH}

There are a vast number of applications of payload directed control systems involving UAVs in the literature, and a comprehensive summary is beyond the scope of this paper. This section presents a summary of few select applications that have relevance to current PDF research directions.

In [1] and [2] vision systems are used to extract road centerlines. Here the road identification process is achieved through image processing that exploits the linear or locally linear nature of roadways. Once the road center line is extracted, the tracking problem is reduced to a simple path following problem. The traditional approach to this problem is to formulate an inner loop controller using linear control theory and design an outer loop controller that uses the desired path to specify the desired bank angle or lateral acceleration and, in some instances, altitude (this is usually constant for the entire mission). In [1] several nonlinear control laws are proposed for solving this problem; interesting control results are also found in [3], [4], [5], and [6]. Additionally, similar research tracking rivers and shorelines is presented in [7] and [8]. An approach for tracking a ground vehicle is presented in [9]. In [10], payload directed flight for fixed-winged aircraft is concerned with having a UAV serve as a communication repeater in a larger communication network. To achieve this, the UAV orbits a radio or communication ground source maintaining a fixed signal-to-noise ratio (SNR) with that source. The problem is strongly analogous to the contour following problem with in-situ sensors. Presented in [10] is a traditional PID based control law formulated around the SNR error between time steps. This formulation, as demonstrated through simulation, will cause the UAV to spiral towards the specified contour (specified SNR value) and remain there once it is acquired. However, implicit in the formulation of this control law is the assumption that the SNR field is monotonic. In many of the cases already presented, this assumption was not applicable and necessitated the development of behavioral approaches.

The re-tasking problem for UASs has also been explored in the literature. In [11] a list of targets to service is treated as a Traveling Salesman Problem. The problem of incorporating vehicle dynamics is achieved by solving the Traveling Salesman Problem using heuristics from traditional combinatorial optimization and then alternating the paths between targets as linear paths and minimum Dubins paths
[12]. Several competing approaches using Dubins minimum paths are also available in [11],[13], [14], and [15].

In [16], the notion of effectively planning given a sensor swath was explored. In this work a UAS has a downward facing camera, which in turn has a certain field of view. The problem is to determine the optimal tour through the targets such that all targets are observed. Unlike the TSP problem where the UAS passes through all the targets, this is a case where it is only necessary for the targets to pass through the sensor's field of view. To solve this problem the UAS was modeled as a Dubins car with a discrete set of inputs $u$ operating over a finite $\Delta t$. The operational space for the UAS was then explored using this model and the learning $A^{*}$ algorithm operating with an admissible heuristic. A similar problem as this was also considered in [17]. Here, however, the operational space is explored using a probabilistic planning approach based on the RapidlyExploring Random Root Tree algorithm, which is thoroughly discussed in [18].

Some of the research in the vein of payload directed flight for fixed-winged vehicles is focused on searching for targets with an unknown location. One example in [19] is probabilistic in nature and involves selecting a search space, discretizing that search space as a grid of cell locations, applying a probability that a target is in a given cell, and identifying the optimal path (in a probabilistic sense) for identifying targets. The research in this field is currently focused on optimal searching with multiple UAVs and optimizing the target identification or mapping ability over many vehicles [20].

\section{Proposed Architectures for Payload Directed Flight Control}

Payload Directed Flight investigates methods for closing control loops around payload sensors, and there are numerous methods used in the literature that accomplish this. Architectures and frameworks are varied in the literature, often suited to fit a particular set of requirements. Architectures are broken into layered functional component-blocks, where 'higher' level blocks deal with increasing complexity and decreasing frequency response rates. The functional decomposition of the system is also highly dependent on the research; research into higher level behavioral algorithms may group lower-level functions into a single block, whereas more fundamental control research may further decompose the lower level control systems into several blocks.

The time response for loop closure can be categorized based on the frequency time response needed for closed-loop system response, as similar to the following categorizations proposed in [21].

1. Direct Closed Loop Sensor Feedback: At the highest frequency update, many requirements call for direct 
closed loop sensor feedback, where control must base off sensor input in a continuous time fashion with a short time period. Such situations as tracking or pointing is a requirement for many missions (e.g., forest fire monitoring for small UAVs [22], carbon flux monitoring [23], or autonomous plume tracking [24]). Methods of feedback control design, such as optimal and robust control, are used to design controllers that weigh benefits of increased science returns with other objectives [21].

2. Tactical Planning: Certain situations call for tactical planning, where current objectives are traded for the possibility of future objectives. For instance maintaining a certain altitude when passing over an object, momentarily sacrificing science return at the current time in order to reposition the aircraft to increase return in the near future. Methods such as predictive control and data-based model prediction have been proposed [21].

3. Strategic Planning: Strategic planning may be necessary to compute trajectories that will position the aircraft and sensors over targets based on previous sensor readings. For instance, based on previous passes over an object, it may be necessary to conduct another pass over an area at a different altitude when sensor readings were insufficient [21][22], or in response to new objectives uploaded by a human operator [25] such as re-tasking requirements for science missions [26][24] or forest fire missions [27]. The system must revise its plan to account for previous and future goals [21].

4. Contingency Planning: Certain scenarios may call for a level of contingency planning. For instance, smoke may obscure sensor instruments, excess aircraft jitter may degrade sensor reading, high wind conditions may cause the aircraft fuel burn rate to be higher than expected between waypoints, or communication links may fail. In these cases, the goals of the system have not changed, but the system must revise its mission plan based on unexpected conditions [21].

Three-Tiered architectures are used in many systems for high-level decomposition, for instance in [31]. These systems typically decompose the control system into highlevel, mid-level, and low-level functional blocks. High level control functional blocks deal with situational awareness, reactive control, and mode selection. Mid-level controllers handle mode transitions of the lower-level components, as well as providing fault detection algorithms and health monitoring functions. The low-level controller provides stability augmentation and control.

Boskovic (et al) introduce a four-layer architecture where the research deals with autonomous intelligent control [35]. The architecture consists of the following layers:
1. Redundancy Management Layer that consists of the online Failure Detection and Identification and robust feedback Adaptive Reconfigurable Controller;

2. Autonomous Trajectory Generation layer whose role is to fit feasible trajectories through the desired waypoints in real time;

3. Autonomous Path Planning layer that generates waypoints on-line in response to a dynamically changing environment; and

4. Autonomous Decision Making layer whose role is to assess the available control authority after failures, and make mission related decisions in near-real time.

Mathematical and computational frameworks have been recently introduced in the literature, which wrap a consistent framework around multiple approaches. For instance, the architecture in [45] introduces a computational framework that addresses trajectory optimization of higher-order systems with general nonlinear constraints.

The following architecture was proposed in [43], which is focused largely on path generation in response to an external source of waypoints:

1. Waypoint Path Planner (WPP): The WPP computes a proposed vehicle route, which is planned without regard for the dynamic constraints on the vehicle. This simplification ensures it is easier to handle dynamic constraints

2. Dynamic Trajectory Smoother (DTS): The DTS applies kinematic constraints to the paths and plans generated by the WPP. The plan is refined through kinematic feasibility.

3. Trajectory Tracker (TT): The trajectory tracker generates uses the kinematically corrected plan from the DTS and computes a feasible state trajectory that can be followed by a standard autopilot.

4. Low-level Autopilot Compensator (LAC): The LAC provides a standard autopilot implementation to follow the trajectory computed from the TT.

The architecture proposed in [25] outlines an Intelligent Autonomous Architecture (IAA) concept in combination with a Collaborative and Coordinated Systems (CCS) component. The IAA combines on-board and ground-based automated systems to control the vehicle and its payload. An autonomous executive performs the basic task of flight and payload operation. The IAA modifies unattainable or conflicting goals in coordination with intelligent specialist systems, which provide goal-directed behavior based on adaptive decision-making models of tactical and strategic 
planning. Plans and execution are modified in direct response to changes in internal conditions, external conditions, and payload related findings. The CCS continuously keeps UAV operators and scientists aware of the current situation onboard the UAV, similar to the Collaborative Information Portal on the Mars Exploration Rover. Participants have a unified interface and access to the internal state of the UAS, including controllers, sensors, payload, and other relevant data sets.

A five-tiered architecture was proposed in [44]. At the highest level, a path planner is responsible for generating paths, which are sets of 3D waypoints without time constraints. A trajectory smoother takes the generated paths and transforms the paths into trajectories, which in the context of this architecture are time parameterized curves; these trajectories define the desired position of the aircraft at specified times throughout the plan. A trajectory tracker component transforms the trajectories and the current state of the aircraft into desired velocity commands, altitude commands, and heading commands, which is then fed into the autopilot system. The autopilot uses sensor feedback to convert the tracking commands into control surface commands. Finally, the aircraft hardware level converts the control surface commands into actual surface deflections.

\section{General Concepts of Operations}

This section outlines general subsonic fixed wing GN\&C operational concepts derived from a survey of ongoing and future missions from the Suborbital Science Program (SSP) under NASA's Science Mission Directorate (SMD). These generic operating concepts are specified to direct aeronautic technology development and innovation in a direction that meets earth science requirements and goals [1][33][39]. Payload directed flight control for suborbital uninhabited platforms provide numerous advantages over manned aircraft and orbital measurements; many benefits are laid out in [26][24][25], thus defining both a need and application area for payload directed flight research. This list summarizes previous efforts that outline future mission concepts and capabilities, as captured in the requirements document for suborbital observations [26], which derived from planning sessions and workshops including the NASA SMD sponsored Suborbital Science Missions of the Future Workshop (SSMFW) in 2004 ([24]-[35]) and the follow-on special sessions at the INTEX site [39].

General requirements for future science missions outlined by the SSP will be roughly divided into three broad categories.

1. Platform Requirements

2. Sensor and Payload Requirements (including communications)

3. General Autonomy Requirements

a. Intelligent Mission Management (IMM) Requirements

b. Payload Directed Guidance, Navigation and Control (GN\&C) Requirements
A dedicated effort to enumerate all specific requirements for payload directed flight, as a subset of general autonomy requirements, has not been conducted in the literature, and is beyond the scope of this paper. "Payload Directed Flight" is directly identified in [26] as a requirement, but does not provide any further elaboration beyond this. The SSMFW sessions [24]-[39] defined candidate mission scenarios, which were used to enumerate and elaborate requirements for platforms and sensor payloads; the publications allude to varying levels of autonomy and intelligence without precise requirement specification at the GN\&C level. Many of the proposed missions outlined in the literature make reference to advanced payload-directed autonomous or semiautonomous flight control modes (e.g., dirty and dangerous plume measurement and tracking [24], identification and tracking of chemical tracers released in cloud systems [24]). IMM enabling concepts have been proposed in the literature [25][21][40][22], from which operational concepts may be drawn.

The workshop members in [24] were divided into six different categories, representing the major focuses of the SSP program. It is interesting to note that each group differed in their inclination or disinclination to include advanced concepts in their mission concepts; such advanced concepts as swarm and hive autonomy, mother/daughter ship concepts, and multiple vehicle coordination were proposed in varying degrees.

NASA has conducted several planning sessions for defining suborbital science missions involving uninhabited aerial systems. The Suborbital Science Missions of the Future Workshop identified over 33 candidate missions [24] [38], with additional mission defined in [39][23][27]. Mission operational concepts and requirements extracted from these missions are varying and wide ranging; analysis in [24] extracts certain trends as general requirements for autonomy across multiple mission. 
Table 1. Science Missions Requirements: Atmospheric Composition and Chemistry

\begin{tabular}{|c|c|c|}
\hline ID & Mission Title and Capabilities & Ref \\
\hline \multirow[t]{7}{*}{1} & Clouds and Aerosols & [24] \\
\hline & Multiple Coordinated Aircraft with Real-Time Command Capabilities & \\
\hline & Stacked formation flying & \\
\hline & Sensor web based vectoring of aircraft & \\
\hline & Precise release of chemical tracers & \\
\hline & Tracking of chemical tracers through cloud system and out-flow region & \\
\hline & Severe weather flying (armored UAV) & \\
\hline \multirow[t]{6}{*}{2} & Stratospheric Ozone & [24] \\
\hline & Lagrangian Sampling & \\
\hline & Race-track sampling & \\
\hline & Spiral Descent Vertical Profiles & \\
\hline & $\begin{array}{l}\text { Isentropic flight }- \text { aircraft adjusts altitude in order to remain on a fixed } \\
\text { isentrope }[\mathrm{q}=\mathrm{T}(\mathrm{P} / 1000) 3.5]\end{array}$ & \\
\hline & Formation flying (alternative scenario) & \\
\hline \multirow[t]{8}{*}{3} & Tropospheric Ozone & [24] \\
\hline & Formation flying with four aircraft & \\
\hline & Near real-time retasking based upon observations from remote sensing platform. & \\
\hline & Following plume events & \\
\hline & Observations of plume synergistic with geostationary platform UV-Vis and IR observations. & \\
\hline & Pre-programmed scenario with retasking & \\
\hline & $\begin{array}{l}\text { Near } \\
\text { based upon observations from remote sensing platform. }\end{array}$ & \\
\hline & Terrain following radar necessary for near-surface in-situ platforms & \\
\hline \multirow[t]{2}{*}{4} & Water Vapor and Total Water & [24] \\
\hline & Spiral Descent Vertical Profiles & \\
\hline
\end{tabular}

Table 2. Science Missions Requirements: Tropospheric

\begin{tabular}{|c|c|c|}
\hline \multirow{3}{*}{$\frac{\text { ID }}{5}$} & Mission Title and Capabilities & Ref \\
\hline & Tracking long-distance pollution & [39] \\
\hline & Plume Tracking & \\
\hline & Long endurance is key & \\
\hline \multirow[t]{5}{*}{6} & Cloud Systems & [39] \\
\hline & Multiple coordinated aircraft & \\
\hline & Stacked formation flying & \\
\hline & Sensor web based vectoring of aircraft & \\
\hline & Identification and Tracking of Cloud Phenomena & \\
\hline \multirow[t]{4}{*}{7} & Long time-scale vertical profiling & [39] \\
\hline & Stacked formation flying (high altitude and low altitude in vertical column) & \\
\hline & Sensor web based vectoring of aircraft & \\
\hline & Spiral Descent/Ascent Vertical Profiles & \\
\hline \multirow[t]{3}{*}{8} & Global 3-D Species & [39] \\
\hline & Global Large-Scale Multiple Vehicle Control Coordination ( 1000 Heterogenious Platforms) & \\
\hline & Vertical Profiling & \\
\hline \multirow[t]{6}{*}{9} & Transport and Chemical Evolution in the Troposphere & [39] \\
\hline & Mothership with Extensive Instrumentation Coordinating Smaller Drones & \\
\hline & Lagrangian Sampling & \\
\hline & Autonomy (mothership) to identify spatial extent of air mass being probed & \\
\hline & Coordinated Control (from Mothership to Drones) & \\
\hline & Closed-loop control around external sensors & \\
\hline 10 & Physical oceanography & [39] \\
\hline
\end{tabular}


Table 3. Science Missions Requirements: Climate Variability and Change

\begin{tabular}{|c|c|c|}
\hline ID & Mission Title and Capabilities & Ref \\
\hline \multirow[t]{4}{*}{11} & Aerosol, Cloud and Precipitation & \\
\hline & Multiple Coordinated Aircraft (planning level, no closed loop control required) & \\
\hline & Waypoint following & \\
\hline & Remote Re-Tasking (altitude leg changes) & \\
\hline \multirow[t]{5}{*}{12} & Glacier and Ice Sheet Dynamics & \\
\hline & Waypoint/Trajectory Following & \\
\hline & Remote Re-Tasking of Altitudes and Location & \\
\hline & Low Altitude Terrain Avoidance & \\
\hline & Precision Navigation (Performance Specs Not Given) & \\
\hline \multirow[t]{5}{*}{13} & Radiation & \\
\hline & Multiple coordinate aircraft (fleet of small UAVs within a vertical column) & \\
\hline & Tight coordinated race track patterns & \\
\hline & Pointing of instrument in NADIR and ZENIGHT for short periods of time & \\
\hline & Tight coordinated upward/downward spirals & \\
\hline
\end{tabular}

Table 4. Science Missions Requirements: Water and Energy Cycles

\begin{tabular}{|c|c|c|}
\hline ID & Mission Title and Capabilities & Ref \\
\hline \multirow[t]{5}{*}{14} & Cloud Properties & [37] \\
\hline & Waypoint Navigation System with Remote Re-Tasking & \\
\hline & Hovering and Circling Search Behaviors & \\
\hline & Look for interesting feature, Release In Situ Samplers & \\
\hline & Mothership/Daughtership & \\
\hline \multirow[t]{8}{*}{15} & River Discharge & [37] \\
\hline & Waypoint Navigation System with Remote Re-Tasking & \\
\hline & Closed-Loop Tracking of Riverbed (LIDAR) & \\
\hline & Low Altitude Terrain Avoidance & \\
\hline & Precision Navigation & \\
\hline & Stacked formation flying & \\
\hline & Closed-Loop Coordinated Control (High Altitude Controls Low Altitude) & \\
\hline & Coordinated Control for Maximum Return & \\
\hline \multirow[t]{7}{*}{16} & Snow-Liquid Water Equivalent & [37] \\
\hline & Waypoint Navigation System with Remote Re-Tasking & \\
\hline & Precision Navigation & \\
\hline & Precision Sensor Pointing & \\
\hline & Search, Identify, Deploy & \\
\hline & Sensor Probe Deployment & \\
\hline & Low Altitude Terrain Avoidance & \\
\hline \multirow[t]{4}{*}{17} & Soil Moisture and Freeze/Thaw States & [37] \\
\hline & Preferred Multiple Aircraft Scenario (Coordinated Flight Control, UAV Sensor Tandems) & \\
\hline & Grid Search and Circling Search Behaviors & \\
\hline & Waypoint Navigation System with Remote Re-Tasking & \\
\hline
\end{tabular}


Table 5. Science Missions Requirements: Carbon Cycle, Ecosystem, and Biogeochemistry

\begin{tabular}{|c|c|c|}
\hline ID & Mission Title and Capabilities & Ref \\
\hline \multirow[t]{3}{*}{18} & Coastal Ocean Observations & {$[34]$} \\
\hline & Near real-time communication and control coordination with underwater vehicles & \\
\hline & Loiter while taking measurements & \\
\hline \multirow[t]{12}{*}{19} & Active Fire, Emissions and Plume Assessment & [34] \\
\hline & Loiter while taking measurements & \\
\hline & Dangerous and dirty plume measurements & \\
\hline & Multiple coordinated aircraft (low plume in situ measurements, higher altitude fire monitoring) & \\
\hline & Instrument deployment/drop & \\
\hline & Plume and Air-Mass tracking and following & \\
\hline & Navigation in extreme temperatures and wind shear & \\
\hline & Collaborative control with other UAVs in swarm & \\
\hline & Terrain Aviodance for low altitude manuevering & \\
\hline & Race-track sampling & \\
\hline & Ascending spiral for vertical measurements & \\
\hline & Stacked coordinated formation flying & \\
\hline 20 & $\mathrm{CO} 2, \mathrm{O} 2$ and Trace Gas Flux Study & {$[34]$} \\
\hline \multirow[t]{4}{*}{21} & Vegetation Structure, Composition \& Canopy Chemistry & {$[34]$} \\
\hline & Waypoint following & \\
\hline & Remote retasking & \\
\hline & Possible: Coordinated control with ground towers and satellites & \\
\hline \multirow[t]{7}{*}{22} & Eddy Covariance Measurements in the Southern Ocean Marine Boundary Layer & {$[23]$} \\
\hline & Swarm of interactive UAVs & \\
\hline & Low Altitude Ocean Navigation & \\
\hline & Obstacle Detection and Avoidance (Nautical Vessels, High Waves, Icebergs) & \\
\hline & Vertical Profile Sampling & \\
\hline & NAS Requirements (See and Avoid, etc.) & \\
\hline & Autonomous Boundary Layer Detection and Tracking & \\
\hline
\end{tabular}

\section{Table 6. Science Missions Requirements: Weather}

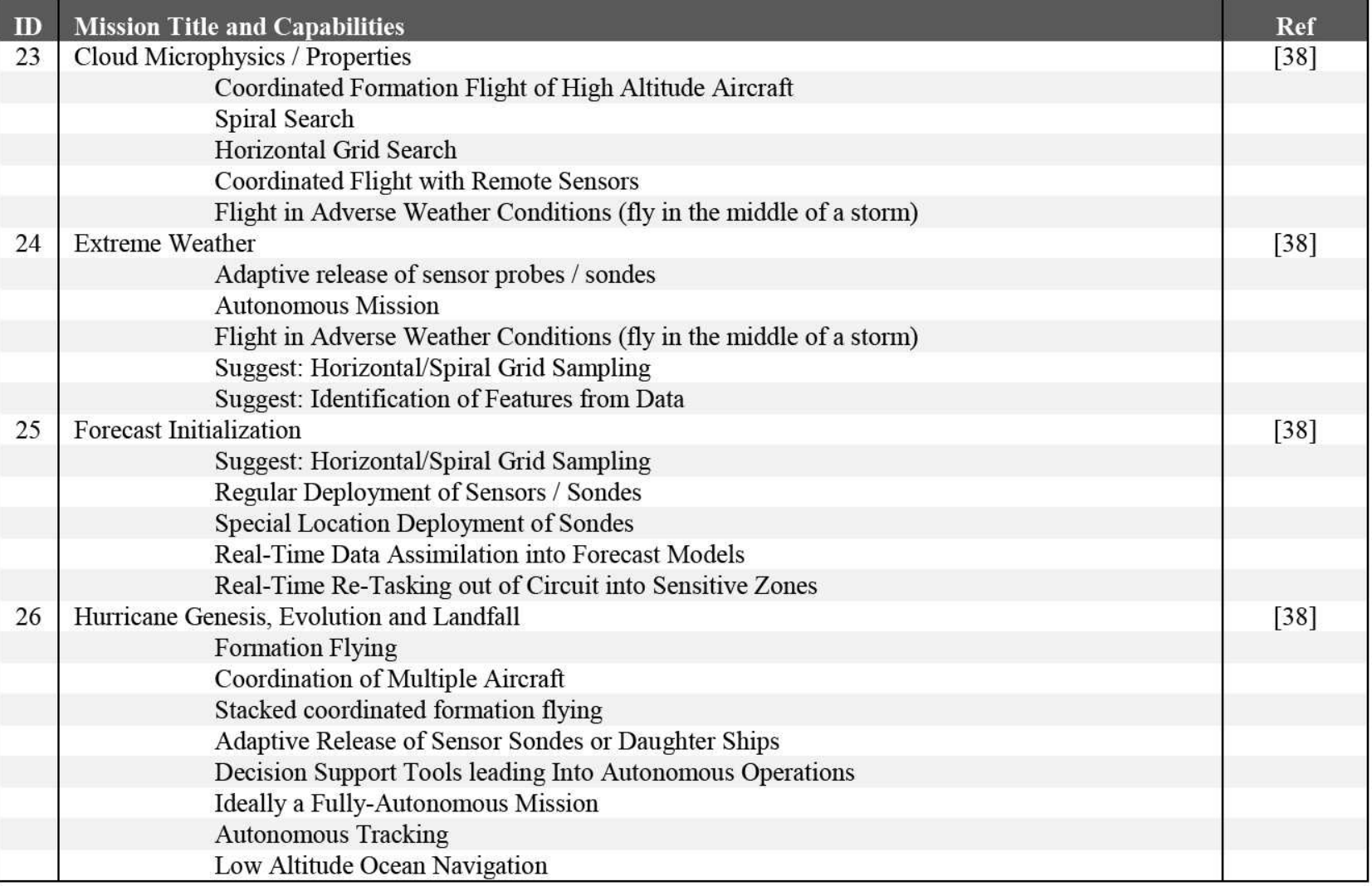


Table 7. Science Missions Requirements: Earth Surface and Interior Structure [35]

\begin{tabular}{|c|c|c|}
\hline ID & Mission Title and Capabilities & Ref \\
\hline \multirow[t]{5}{*}{27} & Surface Deformation & [35] \\
\hline & Precision Navigation ( $+/-5$ meters $)$ & \\
\hline & Precision Pointing of Instruments & \\
\hline & Formation Flying - Collaborative Control & \\
\hline & Fly Long Level Flight Lines in a Grid Pattern, $+/-5$ meters & \\
\hline \multirow[t]{5}{*}{28} & 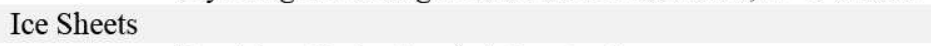 & [35] \\
\hline & Precision Navigation ( $+/-5$ meters $)$ & \\
\hline & Precision Pointing of Instruments & \\
\hline & Formation Flying - Collaborative Control & \\
\hline & Fly Long Level Flight Lines in a Grid Pattern, +/- 5 meters & \\
\hline \multirow[t]{4}{*}{29} & Surface Measurements using Imaging Spectroscopy & {$[35]$} \\
\hline & Precision Navigation $(+/-5$ meters $)$ & \\
\hline & Formation Flying - Collaborative Control & \\
\hline & Fly Long Level Flight Lines in a Grid Pattern, +/- 5 meters & \\
\hline \multirow[t]{3}{*}{30} & Topography using LIDAR & {$[35]$} \\
\hline & Fly Long Level Flight Lines & \\
\hline & Precision Navigation in Corridors & \\
\hline \multirow[t]{3}{*}{31} & Gravitational Acceleration & {$[35]$} \\
\hline & Precision Navigation $(+/-30$ meters $)$ & \\
\hline & Preprogrammed Waypoints and Trajectories & \\
\hline \multirow[t]{2}{*}{32} & International Polar Year & {$[35]$} \\
\hline & Waypoint following & \\
\hline \multirow[t]{5}{*}{33} & Magnetic Fields & {$[35]$} \\
\hline & Collaborative control with other UAVs in swarm & \\
\hline & Tight Coordinated Grid Search & \\
\hline & Formation Flying & \\
\hline & Precision Navigation & \\
\hline 34 & Terrestrial reference frame stability & [35] \\
\hline
\end{tabular}




\section{General ReQuirements}

\section{Req 1.0. Sensor Web Integration}

Almost every scenario requires integration of multiple platforms and payloads - from satellites carrying imagers or radar to subsurface underwater vehicles with optical cameras or water chemistry instruments - in order to accomplish the mission goals. This requirement is mission dependent, and will likely require tactical or strategic planning, for instance, based on real-time data collected and processed and transmitted from multiple remote platforms to an aerial platform within a given time constraint. The mechanism by which this data can be shared is largely an information technology problem, and is the subject of current active research. PDF algorithms must operate over existing communication networks that implement the sensor web. Therefore the science payload guiding PDF implementations should not necessarily be assumed to be integrated on the aircraft of interest.

\section{Req 1.1. Sensor Web Based Vectoring of Aircraft}

High level integration at the outer loop planner and scheduler is required in several missions, such as the Vegetation Structure, Composition and Canopy Chemistry mission in [34], or synergizing plume observations with geostationary platforms and other aerial platforms in the Tropospheric Ozone mission [24]. Lower level integration is a requirement for several missions. A particular sensorweb integration requirement at a lower level comes from the Southern Ocean mission described in [23]. A high altitude aircraft or satellites carrying imaging spectrometers or tunable LIDAR must provide guidance vectors or sensor information to a low altitude aircraft. The low altitude aircraft, carrying gas samplers and nadir and zenith high resolution imaging spectrometers, will utilize this information, along with information from satellites, to attempt to sample locations of maximum carbon flux

\section{Req 1.2. Convergent Modeling and Control of Platforms}

The following possible requirement came from discussions with earth scientists. Given a sensor web integration of multiple platforms, consider the problem of real-time integration of various sensors and payloads into a given model. An example would be to map and model the emission plume of a 1 ha forest fire using optical, active, and gas sampling systems. Models would ingest weather, fuels, and high resolution wind fields to provide probable plume behavior and this would set initial conditions for the flight planner. Processed data from instruments on different aircraft would then be used to update flight paths, and the model could reset initial conditions for follow-on runs. This approach might be used to verify an existing model, or provide data to generate a new model of poorly understood phenomena. The uncertainty in the model is used to vector the various platforms and sensors to specified locations in order to meet the objectives.

Req 2.0. Precision Maneuvering, Navigation and Tracking

Aerial platforms are required to perform navigation tasks and track objectives to various degrees of accuracy. The exact accuracy required is dependent on the specific mission requirements. The accuracy requirement must be used to match missions to particular platforms and suites of avionics. More stringent requirements on navigational accuracy may place requirements on the navigational instrumentation. Certain platforms may not have sufficient controllability to meet the mission required tolerances.

\section{Req 2.1. General Programmable Autopilot}

General capabilities for most missions require an autopilot with autonomous flight capability. A minimum requirement is rough tracking between waypoints, while some missions require programmable trajectories; for instance, the Surface Deformation mission [35] require high accuracy trajectory tracking to within $+/-5$ meters.

\section{Req 2.2. Remote Re-Tasking}

Per requirements in Req 2.1, all functionality of the general programmable autopilot system must be fully accessible through over the horizon communication links. At any point in the mission, operators must be allowed to reprogram the autopilot with new waypoints or trajectories.

\section{Req 2.3. Lagrangian Sampling}

The influence of the sampling time on processes which are under the influence of atmospheric diffusion, such as plume sampling, can be accounted for using Lagrangian sampling, in which a volume is sampled over a time interval Lambda at a fixed downwind distance (travel time) from the source, using a simple modification to Taylor's equation for absolute diffusion. Direct application of this general approach is too complex to apply in practice, and simplifications and approximations have been developed for application [41]. The guidance algorithms for implementation must be synchronized with the payload for accurate sample control, as well as the onboard sensors suite for accurate data collection that is synchronized with the onboard sensor suite.

\section{Req 2.4. Race-Track and Grid Search Patterns}

Reprogrammed sampling at periodic $2 \mathrm{D}$ intervals provides a simpler sampling requirement than in Req 2.3. In this scenario, depending on the accuracy needed, the general autopilot requirement of Req 2.1 may be sufficient for navigation. Onboard avionics and navigation sensors must be synchronized with the payload for accurate sample control. 
Req 2.5. Vertical Profiling through Spiral Ascent and Descent in Vertical Column

Vertical profiling is a requirement for several missions that require sampling gas constituents or measuring radiative properties within a column of the atmosphere. General ascending and descending modes must be synchronized with the payload for accurate sample control, as well as the onboard sensors suite for accurate data collection that is synchronized with the onboard sensor suite.

\section{Req 2.6. Tracking of Chemical Tracer through Cloud System and Outflow Region}

The requirement for tracking a chemical tracer comes from the Cloud and Aerosol mission outlined in [24]. A tracer chemical is released in the boundary layer near the bottom of the in-flow region and trace its transport through the cloud system to the out-flow region. Proposed tracer chemicals include hydrocarbons, formaldehyde (1), HNO3, NOy*, $\mathrm{CO}_{2} *, \mathrm{CO}^{*}, \mathrm{HCl}, \mathrm{CH} 3 \mathrm{I}^{*}$, and sulfur species (e.g., $\mathrm{H} 2 \mathrm{SO} 4, \mathrm{SO} 2$ ). The controller must position the aircraft to sample the species with onboard sensors, and then control the vehicle to accomplish two simultaneous objectives: the controller must position the vehicle such that the sensors to track the target, and the controller must navigate to track the phenomena. This maneuvering must be performed in synchronization with sensors for accurate data collection that is synchronized with the onboard sensor suite.

\section{Req 2.7. Isentropic Flight Tracking}

The Strospheric Ozone mission in [24] requires isentropic flight. In this control scenario, the aircraft must adjust altitude (presumably on an arbitrary navigation path) in order to remain on a fixed isentrope. This maneuvering must be performed as a function of concern upon airborne ozone instruments and ozone column concentration maps from orbital assets such as OMI on AURA, and model estimate. Each data source would be weighted differently based upon known biases or other uncertainties in the data products and as a function of their particular vantage point.

\section{Req 2.8. Autonomous Boundary Layer Detection and Tracking}

Several science missions, such as the Southern Oceans mission in [23] and the Cloud and Aerosol mission outlined in [24], require a vehicle to autonomously detect a surface boundary layer via pressure, temperature, and chemical composition, and track this layer. This requirement may be seen as similar to isentropic flight requirements in Req 2.7.

\section{Req 2.9. Plume Air-Mass Following/Tracking}

The aircraft must be able to identify and track a plume of water vapor or other constituent undergoing atmospheric processes, including diffusion, air mass translation, etc. This requirement stems from several missions, including the Tracking Long-Distance Pollution [39] mission and the Active Fire Emission and Plume Assessment mission [34]. The vehicle must position the aircraft to sample the species with onboard sensors, and guide the vehicle to (1) position the sensors to track the target, and (2) navigate while positioning to track the phenomena. This maneuvering must be performed in synchronization with sensors for accurate data collection that is synchronized with the onboard sensor suite.

\section{Req 2.10. Low Altitude Terrain Altitude Following}

Low-altitude maneuvering is required for many types of missions. In this control scenario, the aircraft must adjust altitude (presumably on an arbitrary navigation path) in order to remain on a fixed altitude from the terrain. Depending on the altitude and terrain characteristics, this may require a mixture of low-level control fixed around an altimeter (such as a LIDAR or RADAR sensor) with strategic and tactical planning to avoid obstacles such as building, trees, mountains, etc.

Req 2.11. Low Altitude Terrain Avoidance, 3D Maneuvering Generally, this requirement requires the planner to be able to compute new trajectories with an arbitrary number and type of constraints. This requirement is similar to Req 2.10, but in a 3D navigational context (rather than just altitude adjustments). This may require computing new trajectories given the constraints of pointing for a particular sensor while avoiding obstacles. This requirement requires the planner to handle pre-specified as well as newly added obstacles.

\section{Req 2.12. Low Altitude Terrain Feature Following and Tracking}

This requirement requires the aircraft to follow a particular ground feature, such as river outwashes, while payloads are constraining the position of the aircraft in some manner. Generally, the trajectory planner and tracker must guide the aircraft along a feature being identified in real time by an optical, Radar, or LIDAR, under arbitrary constraints which may be provided by the payload/sensor or other sources. For instance, the aircraft may be constrained to point a body-fixed sensor to track a feature while avoiding obstacles, or fly straight-and-level at a desired altitude above ground level over undulating terrain.

\section{Req 2.13. Low Altitude Urban Avoidance}

This requirement includes Req 2.11 and Req 2.12, but requires consideration of the characteristics of urban environments. This includes constraints for safety, safe flight termination zones, and sensors that can work on manmade obstacles such as thin power-lines.

\section{Req 2.14. Low Altitude Ocean Navigation and Avoidance}

This requirement is similar to Req 2.11 through Req 2.13. Ocean navigation requires altitude tracking and obstacle avoidance. Such obstacles include large waves, nautical vessels, and icebergs.

\section{Req 2.15. Maneuvering in Extreme Weather Conditions}

Several missions require maneuvering in severe wind, weather, and environmental conditions, such as surviving 
hurricane conditions in the Extreme Weather mission and the Hurricane Genesis, Evolution, and Landfall mission [38], Dirty and Dangerous Plume Measurements [34], or flight over active forest fires with dangerous and turbulent wind shear due to extreme thermal gradients [34]. The exact accuracy needed depends on the particular mission. However, general approaches for robust control formulation and disturbance rejection may be critical for successful completion of these missions. Information from other aircraft, dropsondes, sonobuoys, and satellites will be needed in addition to onboard pressure, temperature, humidity and LIDAR or Radar altimeters.

\section{Req 2.16. Loitering while Sensor/Payload Data Collecting}

Several missions require loitering while collecting sensor and payload operations. Loitering is an endurance phase of flight that often seeks to maximize time over a given target. This requires minimal energy maneuvering with constraints of fielding the payload sensors, which serves to maximize available time over target during this phase of the mission. Optical cues could be incorporated for imagers, while aircraft state data (GPS, INS) and an altimeter could be used for gas sampling.

\section{Req 2.17. Dangerous and Dirty Plume Measurements}

Flight through dirty plumes is highly problematic. For instance, air breathing engines can stall due to aerosol contamination and oxygen starvation. Sensors, such as those designed for atmospheric sampling, may become inoperable due to an accumulation of residue and dirty contaminants. Strong updrafts and turbulent wind conditions can occur in these regions in the presence of extreme thermal gradients. Additional navigation and communication sensors may become inoperable due to flight in this environment. The requirement for measurements in dangerous and dirty plumes must account for these issues. See section 1.2 above for a mission description.

\section{Req 3.0. Multiple Vehicle Coordination}

\section{Req 3.1. General Formation Flying Requirements}

A survey of the variety of techniques for implementing formation flight is well beyond the scope of this document. Techniques include simple swarming algorithms, follow the leader, energy and entropy techniques, distributed control approaches, etc.. Each of these approaches requires their own set of requirements, including communication hardware, networking requirements, specific sensors which must point and track other aircraft, etc. Precise specification of these requirements is dependent on the approach chosen.

\section{Req 3.2. Precision Sub-Meter Precision Flying}

The requirement for precision, sub-meter formation flying was specified as a 'miracle' technology in [24] that would be of great value to the scientific community. The approaches to solve this problem may include highly accurate state estimation sensors onboard formation flying aircraft, with low latency point-to-point communication, or dedicated high-precision sensors for ship to ship distance and vector measurements. The avionics system recently implemented on the NASA DFRC G-III in order to fly repeat passes within $10 \mathrm{~m}$ of previous passes is the state of the art for that class of aircraft. This system was implemented to support interferometry from the UAVSAR.

\section{Req 3.3. Tightly Coordinated Race-Track/Grid-Pattern Sampling}

Several missions, including the radiation mission in [38], specified formation flying aircraft performing precision coordinated race-track and grid-search patterns. The accuracy needed depends on the particular mission requirements. This accuracy requirement would be needed to determine the approach used for coordination.

\section{Req 3.4. Vector-Based Formation Flying}

Several mission scenarios require vector-based formations. There are several techniques for this approach, including reliance on high-accuracy sensors and navigational aids, combined with real-time communication between aircraft. Other approaches include vision-based navigation, and RADAR based sensing of surrounding aircraft. Precise specification of these requirements is dependent on the approach chosen.

\section{Req 3.5. Stacked Formation Maneuvers and Flying}

Stacked formation flying requires performing coordinated maneuvers, such as the requirements in Req 2.0, while coordinating at a low-level control, tactical, or strategic level with other vehicles in the team that are spaced at vertical intervals of a specific altitude-delta. As with other formation flying requirements, precise specification of these requirements is dependent on the approach chosen.

\section{Req 3.6. Stacked Coordinated Closed-Loop Control}

Stacked control coordination requires vehicles at different altitudes to close the loop around remote sensors and data sets, as is required in missions such as the River Discharge mission [37]. This application was demonstrated in the Maldives in 2007 by Ramanathan (Science citation) using a Mantas below, within, and above clouds in the same column to within a few meters.

\section{Req 3.7. Autonomous Coordinated Control for Maximum Data Return}

These requirements, such as found in the River Discharge mission [37] and Southern Ocean mission [23], stem from generic statements about the desire for a team of multiple unmanned platforms to share information and maximize data return given the available resources. The specific requirements will depend on the approaches used to accomplish this goal.

\section{Req 3.8. Coordinated Spiral Descent/Ascent in Vertical Column}

This requirement is the same as Req 3.5. However, the requirement for vertical profiling or vertical traverse in a 
vertical column is prevalent enough and unique enough that a specific formulation for this problem may be warranted, so it is included as its own requirement. As with other formation flying requirements, precise specification of these requirements is dependent on the approach chosen.

\section{Req 3.9. Interactive UAV Swarms}

Various swarming algorithms have been proposed and implemented in the literature. The use of UAV swarms is referenced in the Southern Oceans mission in [23]. Many algorithm implementations require agent-based UAV's to be aware of the location of other UAV's either through specific payload sensors or inter agent communication to share sensor information. Agent to agent communication requires efficient utilization of communication bandwidth. Alternatively, advanced networking approaches such as introduced in [46] can be utilized to optimize network usage.

\section{Req 3.10. UAV Sensor Tandems}

The sensor tandem requirement (from such missions as the Soil Moisture and Freeze/Thaw States mission in [37]) requires a team of UAVs with identical sensors to be deployed in a area and coordinate their traversal to maximize coverage of a specified over time, while minimizing overlap. This general requirement may be modified to some extent, given the requirements of the mission. For instance, teams may be required to overlap sensor coverage within a given time window, in order to correlate sensor readings.

\section{Req 4.0. Sensor and Daughtership Deployment}

The ability for aircraft to deploy sensors, sondes, bouys, daughterships, and small expendable platforms was identified as a 'miracle' technology in [24] that would be of great value to the scientific community. Such a system has been demonstrated by NRL in the Finder, which is deployed from the wing of Predator-class aircraft. Global Hawk flights over hurricanes beginning in 2010 will likely deliver dropsondes and so models of hurricane , GOES imagery, weather data from P-3 Hurricane Hunters, and low altitude UAS will likely be used to optimize deployment.

\section{Req 4.1. Autonomous Search, Identify, Deploy}

This requirement is specified in missions such as the SnowLiquid Water Equivalent mission [37]. The unmanned autonomous platform must direct onboard payload sensors to search a particular area for a specified feature. The vehicle must deploy expendable or reusable sensor payloads at precise locations. Deployment may occur immediately on recognition, or after based on a certain criteria. Once deployed, the vehicles must act as data collectors and relays for the deployed sensors.

\section{Req 4.2. Precision Release of Tracer Chemical Agent Tracer}

The requirement for tracking a chemical tracer comes from the Cloud and Aerosol mission outlined in [24]. This requirement is the precursor to the tracer tracking in Req 2.6. In this particular mission, a tracer chemical is released in the boundary layer near the bottom of the in-flow region and its transport is traced through the cloud system to the out-flow region. The platform must determine the precise location for the chemical release. This might be through sampling, tracking around a sensor, etc. Once determined, the platform must reach a particular position/orientation within a time constraint, and release the agent at this position. The release actuation must be synchronized with onboard sensors and payloads.

\section{Req 4.3. Mother/Daughtership Deployment and Control} The Transport and Chemical Evolution in the Troposphere mission [39] species coordinated control of a multiple UAV system from a single mothership. The mothership contains extensive remote instrumentation, and will deploy multiple drones over a target area. The mothership will provide communications, command and control of the drones with in situ instrumentation flying above and below. This particular mission specification may be overly specified at to the particulars of the mission (for instance, the control may be agent-based peer-to-peer, rather than a master-slave relationship), but gives a general and very future looking mission scenario.

\section{Req 4.4. Mother/Daughtership Redocking}

The ability for a mothership to deploy smaller vehicles and then have these autonomous vehicle redock with the mothership was specified as a 'miracle' technology in [24] that would be of great value to the scientific community, Current state of the art is insufficient to pursue a mission which requires an aircraft to consistently deploy and retrieve smaller aircraft in a single mission. Robust and reliable redocking is an area of current research, and specific requirements would be drawn from the specific approach used.

\section{Conclusion AND Future Research}

Next generation aviation flight control concepts require autonomous and intelligent control system architectures that close control loops directly around payload sensors in manner more integrated and cohesive that in traditional autopilot modes. This paper has presented a sampling of various requirements and operating concepts for payload directed flight concepts. Presently, flight systems designed to perform payload-centric maneuvers require preconstructed procedures and special hand-tuned guidance modes. To enable intelligent maneuvering via strong coupling between the goals of payload-directed flight and the autopilot functions, there exists a need to rethink traditional autopilot design and function. Continuing research into payload directed flight examines sensor and payload-centric autopilot modes, architectures, and algorithms that provide layers of intelligent guidance, navigation and control for flight vehicles to achieve mission goals related to the payload sensors, taking into account 


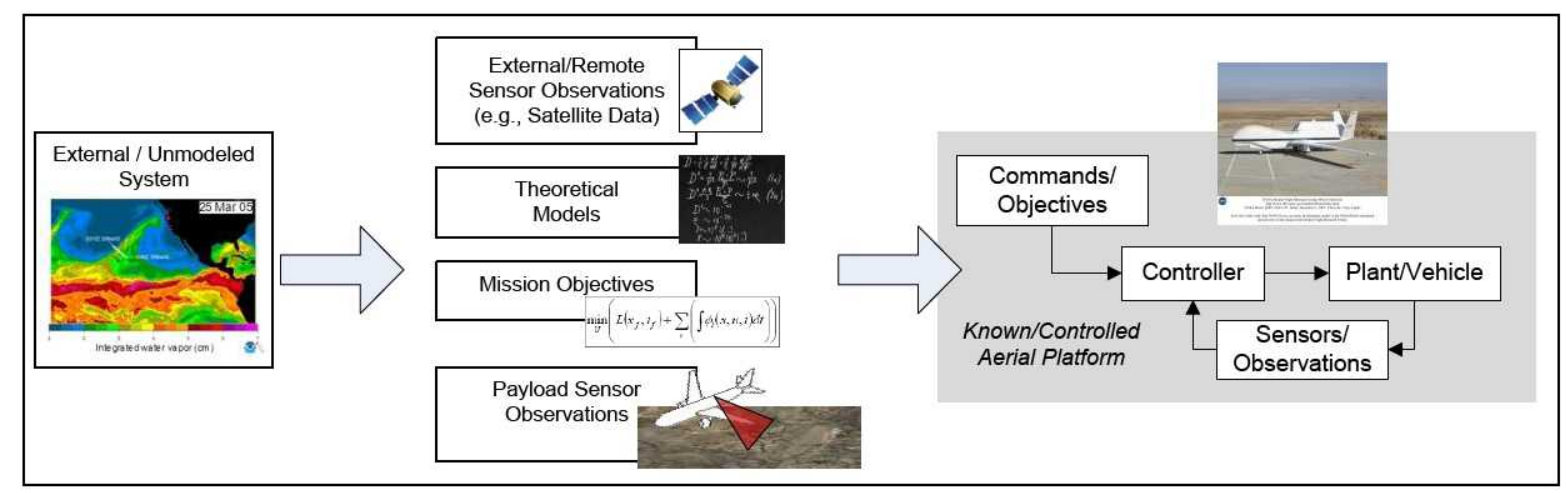

Figure 1 - A Prototypical Payload Directed Flight Problem

various constraints such as the performance limitations of the aircraft, target tracking and estimation, obstacle avoidance, and constraint satisfaction.

A central problem to address in payload directed flight is to control a known and controllable plant interacting with an external system based on payload and sensor data feedback that gives partial observation and understanding of the external system, to satisfy mission objectives and constraints on the combined system. This is shown conceptually in the block diagram in Figure 1, where a controllable system is coupled with an external system which may be unmodeled or poorly modeled for various reasons. These reasons may include complexity, uncertainty, lack of observability from sensor to state, the size of the external system's state may overwhelm computational and modeling resources, or lack of available data to generate a model. A suite of sensors provide some set of observations into the system, and a set of mission objectives are defined concerning the combined system. The PDF research objectives seek methods, tools, and techniques for designing controllers around these blocks to ensure the combined system meets mission objective under varying constraints.

Current payload directed flight research at NASA Ames Research Center focuses on near-optimal trajectory generation and flight control under varying constraints in a highly dynamic environment, autonomous feature detection and estimation, and modeless autopilot design concepts for multi-objective system control. Application of this research is targeted towards increasing capabilities, performance, and efficiency in the execution of missions that require payloaddirected and target-directed maneuvering, towards the goal of proliferation of this technology throughout the manned and unmanned aviation sector.

\section{REFERENCES}

[1] E. Frew, T. Mcgee, Z. W. Kim, X. Xiao, S. Jackson, M. Morimoto, S. Rathinam, J. Padial, R. Sengupta, "VisionBased Road-Following Using a Small Autonomous Aircraft," Proc. IEEE Aerospace Conference, 2004.

[2] S. Rathinam, Z. Kim, A. Soghikian, R. Sengupta"Vision Based Following of Locally Linear Structures Using an Unmanned Aerial Vehicle", Proc. 44th IEEE Conference on Decision and Control, 2005.

[3] S. Park, J. Deyst, and J. P. How, "A New Nonlinear Guidance Logic for Trajectory Tracking", AIAA Guidance, Navigation, and Control Conference, 2004.

[4] H. Kim, D. H. Shim, S. Sastry, "Nonlinear Model Predictive Tracking Control for Rotorcraft-based Unmanned Aerial Vehicles," Proc. of the American Control Confer., 2002.

[5] R. Frezza, G. Picci, S. Soatto, "A Langragian Formulation of Nonholonomic Path Following," The Confluence of Vision and Control, Springer Verlag, 1998.

[6] A. Matsuura, S. Suzuki, M. Kono, A. Sakaguchi, "Lateral Guidance Control of UAV using Feedback Error Learning," AIAA Infotech@Aerospace, Rohnert Park, CA 2007.

[7] S. Rathinam, P. Almeida, Z. W. Kim, S. Jackson, A Tinka, W. Grossman, R. Sengupta, "Autonomous Searching and Tracking of a River Using an UAV", Proc. of the American Control Conference, 2007.

[8] P. Baker, A. Kahn, B. Kamgar-Parsi, J. Kellogg, "Optical Guidance for UAV Following of Shorelines," AIAA Guidance, Navigation, and Control Conf., 2007.

[9] J. Lee, et. al. , "Strategies of Path-Planning for a UAV to Track a Ground Vehicle," AINS Conf., 2003. 
[10] C. Dixon, E. Frew, B. Argrow, "Radio Leashing of an Unmanned Aircraft," AIAA Infotech@Aerospace, VA, Arlington, VA 2005.

[11] K. Savla, E. Frazzoli, F. Bullo, "On the point-to-point and Traveling Salesperson Problems for Dubins' Vehicle," in American Control Conference, 2005.

[12]L. E. Dubins, On Curves of Minimial Length with a Constraint on Average Curvature and with Prescribed initial and Terminal Positions and Tangents, American Journal of Mathematics, Vol 79, 1957.

[13]X. Ma, D. A. Castanon, "Receding Horizon Planning for Dubins Traveling Salesman Problems," Proc. IEEE Conf. on Decision and Control, 2006.

[14] T. Fraichard, A. Scheuer, "From Reeds and Shepp's to Continuous-Curvature Paths," IEEE Transactions on Robotics, Vol. 20, No. 6, December 2004.

[15] A. Scheuer, Ch. Laugier, "Planning Sub-Optimal and Continuous-Curvature Paths for Car-Like Robots," Proceedings of the 1998 IEEE/RSJ International Conference on Intelligent Robots and Systems, Victoria, B.C., Canada, October 1998.

[16] J. K. Howlett, T. W. Mclain, and M. A. Goodrich, "Learning Real-Time A* Path Planner for Unmanned Air Vehicle Target Sensing," Journal of Aerospace Computing, Information, and Communication, Vol. 3 , March 2006.

[17] J. J. Kehoe, A. S. Watkins, and R. Lind, "Trajectory Generation for Effective Sensing," AIAA Guidance, Navigation, and Control Conference and Exhibit, Hilton Head, SC, 2007.

[18]E. Frazzoli, M. Dahleh, and E. Ferron, "Real-time motion planning for agile autonomous vehicles," in Proceedings of the AIAA Guidance, Navigation, and Control Conference, (Denver, CO), August 2000. AIAA Paper No. AIAA-2000-4056.

[19]L. F. Bertuccelli, J. P. How, "Robust UAV Search for Environments with Imprecise Probability Maps," Proc. IEEE Conf. on Decision and Control, 2005.

[20] Y. Jin, A. Minai, M. Polycarpou, "Cooperative RealTime Search and Task Allocation in UAV Teams," IEEE Conf. on Decision and Control, 2003.

[21]D. Sullivan, et al. Intelligent Mission Management for Uninhabited Aerial Vehicles, SPIE Proceedings Vol. 5661, 22 December 2004
[22] Casbeer, D.W. Beard, R.W. McLain, T.W. SaiMing Li Mehra, R.K. Forest fire monitoring with multiple small UAVs. American Control Conference, 2005. Proceedings of the $2005,3530-3535$ vol. 5

[23] M. Fladeland et al, Eddy flux measurements in the Southern Ocean marine boundary layer using long duration, low-altitude robotic aircraft, NASA Suborbital Science Missions of the Future, March 11, 2005

[24] M. Kurylo, Atmospheric Composition - Science Focus Work Group Products, NASA Suborbital Science Missions of the Future Workshop, July 10-12, 2004, Arlington, VA

[25]M Fladeland et al, A suborbital observation system for measuring carbon flux over land and water, Infotech@Aerospace, 26 - 29 September 2005, Arlington, VA

[26]NASA Earth Science Requirements for Suborbital Observations, NASA Science Mission Directorate, Earth Science Division, June 2007

[27]V. Ambrosia et al. The UAV Western States Fire Mission: Concepts, Plans and Developmental Advancements. AIAA 3rd "Unmanned Unlimited" Technical Conference, Workshop and Exhibit 20 - 23 September 2004, Chicago, Illinois

[28]C. Yuhas, et al. Suborbital Science Missions of the Future Workshop, NASA Suborbital Science Missions of the Future Workshop, July 10-12, 2004, Arlington, VA, http://geo.arc.nasa.gov/uavsuborbital/docs/SSMF Wksp Report v2.0.pdf, Accessed November 11, 2007

[29] S. Schoenung, Suborbital Science Missions of the Future Workshop Summary Report, NASA Suborbital Science Missions of the Future Workshop, July 10-12, 2004, Arlington, VA

[30] S. Schoenung. Intelligent UAV Airborne Science Missions. Infotech@Aerospace. 26 - 29 September 2005, Arlington, Virginia

[31] S L. Wills, S. Kannan, B. Heck, G. Vachtsevanos, C. Restrepo, S. Sander, D. Schrage, J. V. R. Prasad "AN OPEN SOFTWARE INFRASTRUCTURE FOR RECONFIGURABLE CONTROL SYSTEMS“, Georgia Institute of Technology, Atlanta, GA 30332

[32] Wegener, S., et al., "UAV Autonomous Operations for Airborne Science Missions, AIAA $3^{\text {rd }}$ Unmanned Unlimited Technical Conference, Chicago, IL, 2004. 
[33]NASA Report to Committees on Appropriations Regarding Potential Use of Unmanned Aircraft Systems (UAS) for NASA Science Missions, May 8, 2006, available at $\underline{\mathrm{http}: / / \text { suborbital.nasa.gov. }}$

[34]M. Fladeland, Carbon Cycle, Ecosystems, and Biogeochemistry - Science Focus Work Group Products, NASA Suborbital Science Missions of the Future Workshop, July 10-12, 2004, Arlington, VA

[35] J. Boskovic, R. Prasanth, R. Mehra, "A Multi-Layer Autonomous Intelligent Control Architecture for Unmanned Aerial Vehicles", JOURNAL OF AEROSPACE COMPUTING, INFORMATION, AND COMMUNICATION, Vol. 1, December 2004

[36] J. LaBrecque, Earth Surface and Interior StructureScience Focus Work Group Products, NASA Suborbital Science Missions of the Future Workshop, July 10-12, 2004, Arlington, VA

[37] J. Entin, Water and Energy - Science Focus Work Group Products, NASA Suborbital Science Missions of the Future Workshop, July 10-12, 2004, Arlington, VA

[38] R. Hood, Weather - Science Focus Work Group Products, NASA Suborbital Science Missions of the Future Workshop, July 10-12, 2004, Arlington, VA

[39]C Yuhas, Atmospheric Composition (Troposphere) Science Focus Work Group Products, INTEX Special Session of NASA Science Missions of the Future Workshop, July 13-15, 2004, New Hampshire

[40] S. Wegener et al. UAV Autonomous Operations for Airborne Science Missions. AIAA-2004-6416, AIAA 3rd "Unmanned Unlimited" Technical Conference, Workshop and Exhibit, Chicago, Illinois, Sep. 20-23, 2004

[41]R. Eckman, The Influence of the Sampling Time on Diffusion Measurements in the Atmosphere. Thesis (PH.D.)--THE PENNSYLVANIA STATE UNIVERSITY, 1989.Source: Dissertation Abstracts International, Volume: 51-02, Section: B, page: 0799.

[42] J. Sonntag at al. Mission Concepts for Uninhabited Aerial Vehicles in Cryospheric Science Applications. AIAA 2005-6921, Infotech@Aerospace, 26 - 29 September 2005, Arlington, Virginia

[43] Singh, L. et al. "Trajectory Generation for a UAV in Urban Terrain, using Nonlinear MPC", Proceedings of the American Control Conference, Arlington, VA June $25-27,2001$
[44]D. Kingston, R. Beard, T. Mclain, M. Larsen, W. Ren, "Autonomous Vehicle Technologies for Small Fixed Wing UAVs", 2nd AIAA Unmanned Unlimited Systems, Technologies, and Operations Conference, September 2003, San Diego, California, AIAA 2003-6559

[45] T Veeraklaew and S Agrawal, "New Computational Framework for Trajectory Optimization of Higher-Order Dynamic Systems" JOURNAL OF GUIDANCE, CONTROL, AND DYNAMICS, Vol. 24, No. 2, MarchApril 2001

[46]C Ippolito, S Joo, K Al-Ali, Y Yeh, "Flight Testing Polymorphic Control Reconfiguration in an Autonomous UAV with UGV Collaboration", IEEE Aerospace Conference, Big Sky, Montana USA, March 2008.

\section{BIOGRAPHIES}

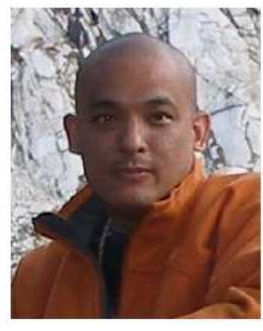

Corey Ippolito is a Research Scientist and Aerospace Engineer at NASA Ames Research Center, task lead for the payload directed flight project, and runs the Exploration Aerial Vehicle lab at Ames. An MSAE from Georgia Tech, Mr. Ippolito is recipient of the NASA Award of Excellence and the NASA Team Achievement Award, with research interests that include vehicle autonomy, control reconfiguration and probabilistic methods in artificial intelligence. He is a contributing member of AIAA and IEEE, with affiliations that include the NASA Haughton-Mars Project, and the NASA Biologically-Inspired Engineering for Exploration Systems for Mars project.

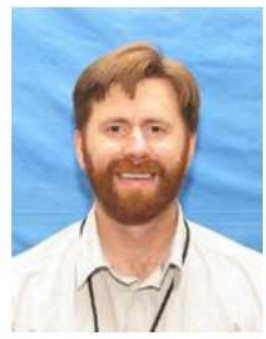

Matthew Fladeland is Manager of the Airborne Science Program Office at NASA Ames Research Center. Mr. Fladeland received an M.S. from the School of Forestry and Environmental Studies at Yale University.

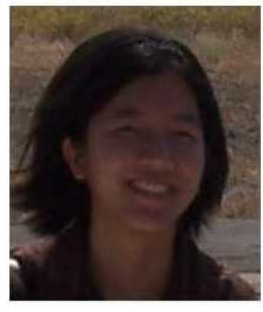

Yoo Hsiu Yeh is a Project Engineer at Carnegie-Mellon University West Coast Campus. She graduated with a B.S.E.E. from Stanford University in 2006, and has been working with the Exploration Aerial Vehicle project at NASA Ames Research Center. She is a member of the IEEE and AIAA. 